\title{
Pseudoaneurysm of the facial artery territory in an odontogenic infection setting
}

\author{
Miguel Jorge Lopes Oliveira ${ }^{1,3} \cdot$ Carina Marques Pereira Semedo ${ }^{1} \cdot$ \\ Maria João Guerreiro Quintela ${ }^{2} \cdot$ Isabel Maria Ribeiro Fragata $^{2}$. \\ Carla Cristina Gonçalves Coelho ${ }^{1} \cdot$ Paulo Jorge Valejo Coelho ${ }^{1}$
}

Received: 28 July 2014/ Accepted: 22 June 2015 / Published online: 30 July 2015

(C) Japanese Society for Oral and Maxillofacial Radiology and Springer Japan 2015

\begin{abstract}
A pseudoaneurysm of the facial artery is a rare event. It results from vessel wall disruption with blood tamponade from the surrounding tissues, and is usually caused by blunt facial trauma, with other etiologies rarely reported. We present two cases of a pseudoaneurysm in the facial artery territory in an odontogenic infection setting, highlighting the importance of computed tomography angiography for diagnosis and hastening treatment by vascular embolization.
\end{abstract}

Keywords Infection - Pseudoaneurysm - Facial artery · Computed tomography angiography $\cdot$ Embolization

\section{Introduction}

An aneurysm is an abnormal and localized dilation of blood vessels, classified as either true or false [1]. A true aneurysm retains all three layers of the blood vessels: intima, media, and adventitia. They are frequently associated with atherosclerosis, but can result from arteritis, infection, or fibromuscular dysplasia [1, 2]. A false aneurysm or pseudoaneurysm results from vessel wall disruption and extravasation of blood into the surrounding tissue, until the pressure in the hematoma exceeds the

Miguel Jorge Lopes Oliveira

sec.maxilo@chlc.min-saude.pt

1 Maxillofacial Surgery Department, Central Lisbon Hospital Center, Lisbon, Portugal

2 Neuroradiology Department, Central Lisbon Hospital Center, Lisbon, Portugal

3 Departamento Cirurgia Maxilofacial, Hospital de S. José, Rua José António Serrano, 1150-199 Lisbon, Portugal arterial pressure, leading to tamponade and clot formation $[3,4]$.

Extracranial head and neck artery pseudoaneurysms are rare. One of the most frequent causes is blunt trauma. Although these vessels tend to be protected by soft tissues, the risk rises as they approach the superficial layers to cross bone structures. The superficial temporal artery, facial artery, and some branches of the internal maxillary artery are the most frequently affected [5-7]. Pseudoaneurysms can develop immediately after a traumatic event, but more usually show delayed occurrence after days, months, or even longer $[7,8]$. There are also reports on mycotic (infective) pseudoaneurysms, which tended to focus predominantly on the carotid arteries [9-11].

Regarding facial artery pseudoaneurysms, besides blunt trauma, there are a few reports presenting them as iatrogenic consequences of molar teeth extraction [12] or craniofacial surgery $[3,13]$. The following two case reports depict facial pseudoaneurysms occurring within an odontogenic infection setting. This article was approved by our institutional health ethics committee (Case No. 215/2015).

\section{Case report 1}

A 42-year-old Caucasian man without cardiovascular risk factors or other systemic illnesses and denying any facial traumatic event was admitted with an 8-day history of a progressively enlarging left submandibular swelling, despite antibiotic cover prescribed at another healthcare institution. Examination on admission found the swelling to be $70 \times 60 \mathrm{~mm}$ in size, tender, and nonpulsatile, with fluctuant purple-bluish areas (Fig. 1a). Intraorally, there was very discrete spontaneous alveolar bleeding at the third 


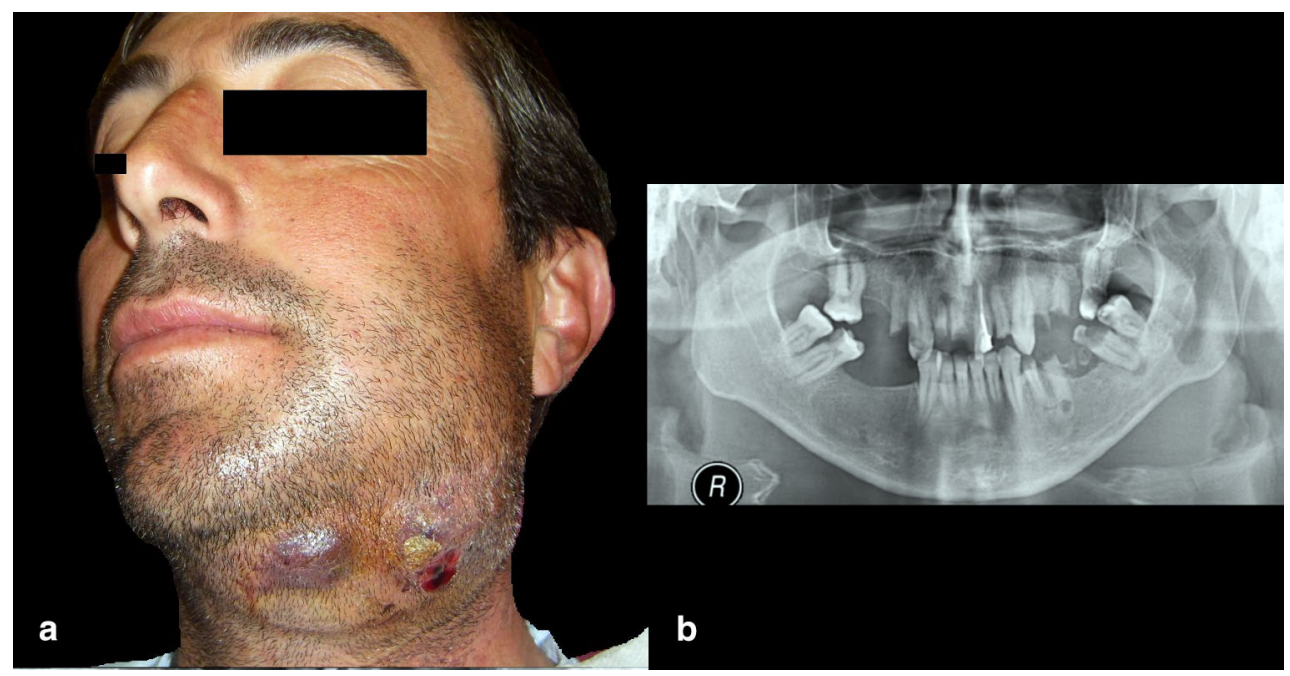

Fig. 1 Case \#1. a Clinical aspect upon admission, showing an enlarged left submandibular swelling. b Panoramic radiograph showing dental caries with periapical osteolysis at the third quadrant

quadrant and obvious precarious dental care. There were no critical signs, such as trismus, airway obstruction, or dysphagia. The clinical features strongly suggested odontogenic cellulitis. Blood tests were unremarkable, with normal hemogram, no raised acute inflammatory markers, and normal values for hepatic integrity, renal function, ionogram, and glucose and lipid profiles.

A noncontrast computed tomography (CT) scan, followed by contrast material injection, showed a spontaneous hyperdensity adjacent to inflamed submandibular tissues along with pus collection (Fig. 2a, b). The inflamed tissues included the masticator space and there was a mass effect on the parapharyngeal and carotid space. A computed tomography angiography (CTA) scan was performed and revealed a $13.5-\times 8.3-\times 10-\mathrm{mm}$ pseudoaneurysm originating from a left facial artery branch (Fig. 2c, d).

Digital subtraction angiography (DSA) was performed, followed by superselective catheterization and successful embolization with cyanoacrylate (Fig. 3a, b). To avoid uncontrolled bleeding, there were no attempts at surgical pus drainage, or even microbiology specimen collection, and treatment relied on intravenous antibiotic therapy (amoxicillin/clavulanate $1.2 \mathrm{~g} \mathrm{q} 8 \mathrm{~h}$ and metronidazole $500 \mathrm{mg} \mathrm{q} 8 \mathrm{~h}$ ) and dental extraction. The patient was discharged 8 days later. At the 4-month follow-up, there were no clinical or imaging signs of either infection or pseudoaneurysm (Fig. 4a, b).

\section{Case report 2}

A 58-year-old Caucasian man was admitted with a 6-day history of a left mandibular firm swelling and trismus. His previous medical history was uneventful. Laboratory tests showed raised inflammatory markers (leukocyte count: $21.70 \times 10^{9} / \mathrm{L}$ with $94.76 \%$ neutrophils; C-reactive protein: $190.6 \mathrm{mg} / \mathrm{L}$ ), without other changes.

A panoramic radiograph showed dental caries on the first and second inferior left molars, with periapical osteolysis (Fig. 5). A noncontrast CT scan at admission showed a phlegmonous infiltration of the left submandibular and masticator spaces, with subtle gaseous areas, suggesting odontogenic cellulitis (Fig. 6a).

Under general anesthesia, urgent surgical drainage and dental extraction were performed. However, unexpected bleeding occurred during extraoral drainage, which was controlled by hemostatic intraoral and extraoral ligatures. Because of this event, no microbiology specimens were collected.

A contrast-enhanced CT scan at 2 days postoperatively suggested a 21-mm pseudoaneurysm on the left facial artery (Fig. 6b), which was immediately confirmed by CTA (Fig. 7a, b). At 5 days postoperatively, the patient underwent conventional angiography (Fig. 8a). The pseudoaneurysm was embolized with cyanoacrylate, with successful aneurism exclusion (Fig. 8b). The patient was 


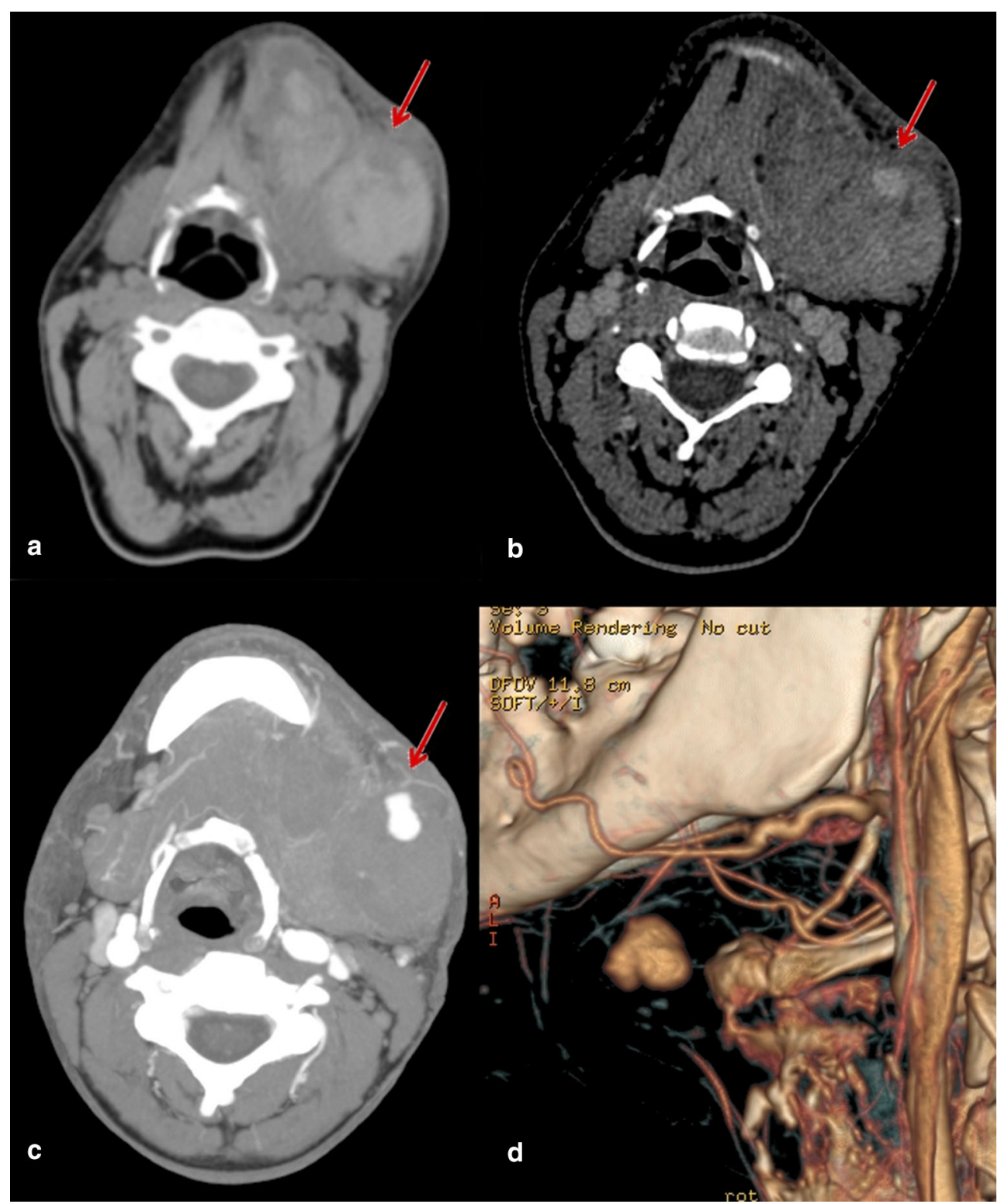

Fig. 2 Case \#1. a, b Noncontrast (a) and contrast-enhanced (b) CT scans upon patient admission, suggesting blood collection (arrow) confined to inflamed submandibular tissues, along with pus collection.

discharged the following day. Antibiotic treatment was the same as for Patient \#1. At the 2-month follow-up, there was only a submandibular residual induration with no other clinical or imaging signs of infection or pseudoaneurysm (Fig. 9a, b; compare with Fig. 7).

\section{Discussion}

In both cases, the diagnosis of odontogenic cellulitis was based on clinical grounds, backed by radiology findings. It is widely accepted that dental infection is one of the main c CTA image demonstrating an enhancing pseudoaneurysm (arrow) in the large mass in the left submandibular area. d CTA with 3D vascular reconstruction

starting points for deep cervicofacial infections in adults [14-16]. The history of the present illness, location of inflammatory signs, and precarious dental care strongly suggested the diagnosis of odontogenic cellulitis for both cases. Imaging confirmed the presence of dental caries, periapical osteolysis, and pus in the submandibular and masticator spaces. However, an unexpected radiology finding of a pseudoaneurysm in the facial artery territory changed the ensuing therapeutic management.

A pseudoaneurysm of the facial artery is extremely uncommon and usually related to trauma, with some rare reported cases of iatrogenic local anesthetic needlestick 

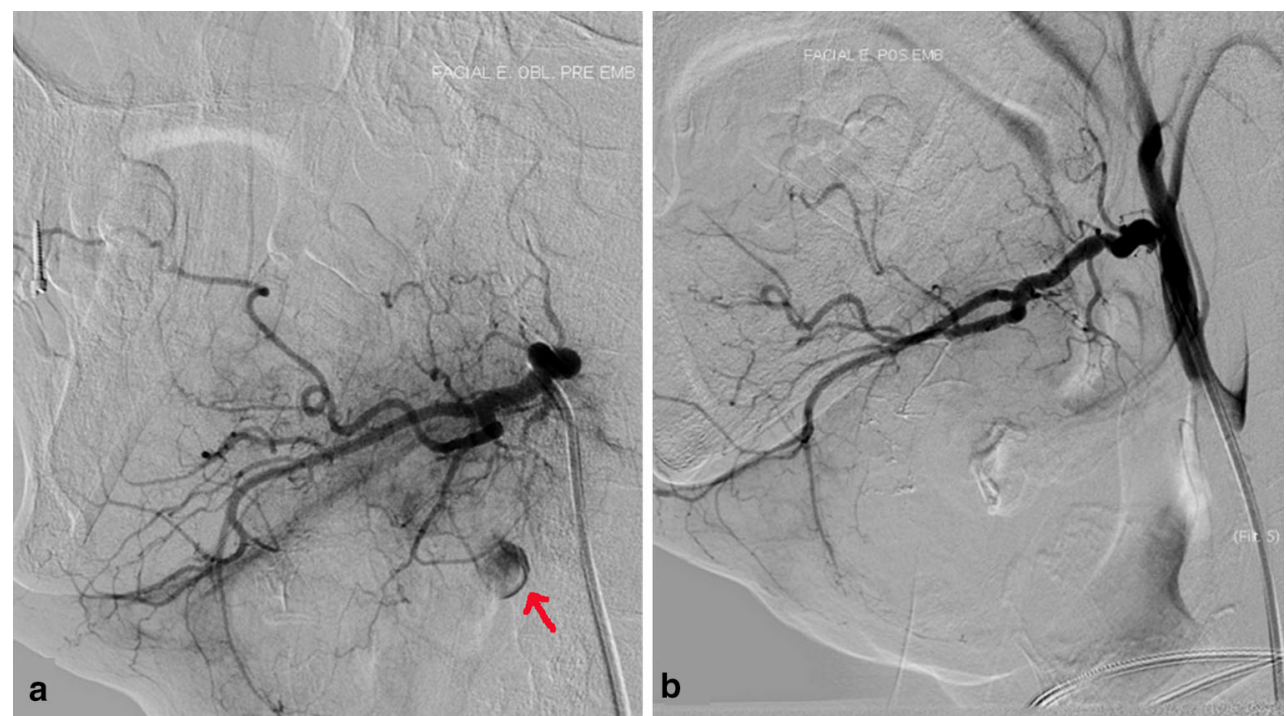

Fig. 3 Case \#1. a Selected DSA image with the catheter tip in the proximal facial artery, showing filling of the pseudoaneurysm in a small branch (arrow). b Selected DSA image after cyanoacrylate embolization, showing successful pseudoaneurysm exclusion
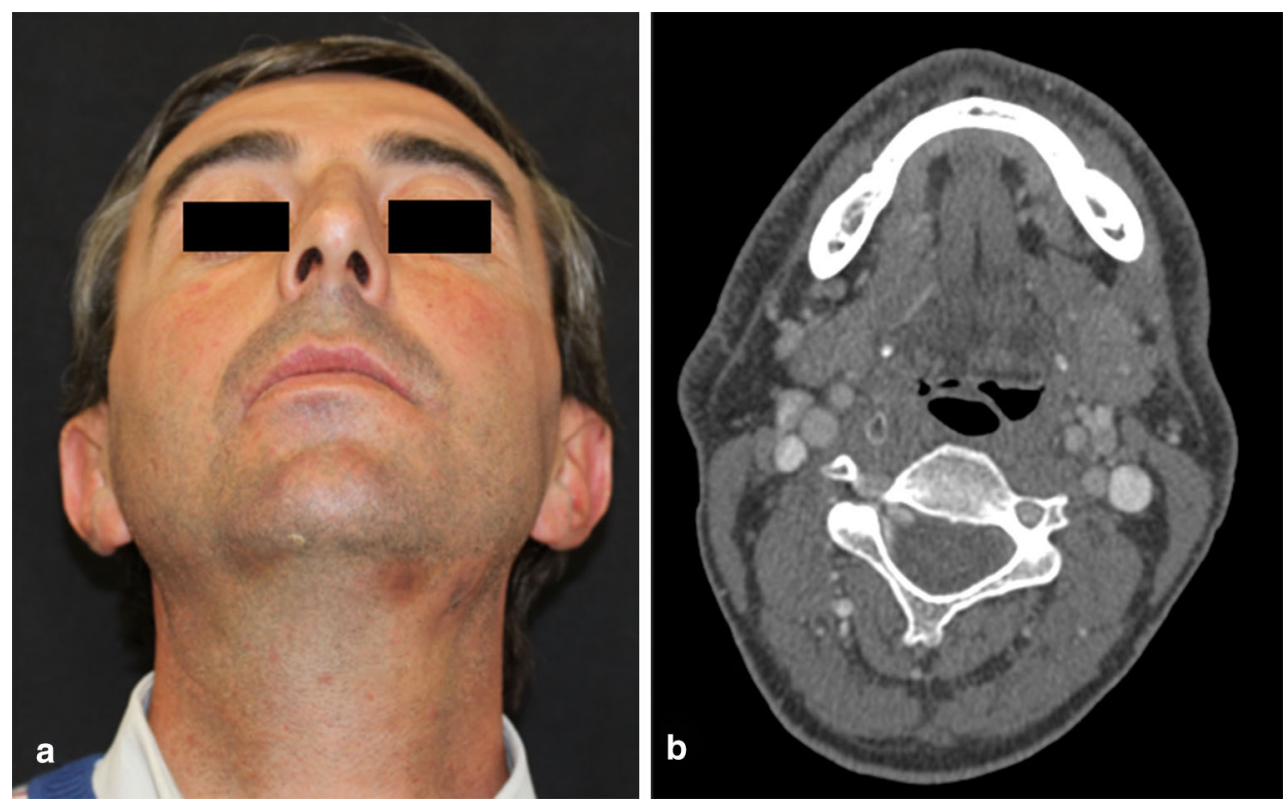

Fig. 4 Case \#1. a Clinical aspect at 4 months after embolization. b CTA image showing aneurysm exclusion, with no evidence of cyanoacrylate, which has probably been reabsorbed

injury, dental extraction [8, 17], orthognathic surgery, and mandibular fracture repair surgery $[3,7,12,18]$. Such pseudoaneurysms can present as a pulsatile mass, with systolic bruits. A differential diagnosis with arteriovenous fistulas should be considered. The latter tend to have a continuous bruit, which is intensified during systole, with murmur transmission $[3,7,8]$.

However, as depicted in the first case report, clinical signs of vascular abnormality can be muffled, in this case by an abscess. Even the purple-bluish fluctuant areas did not raise suspicion, until CT was performed. There were no clinical or laboratory tests suggestive of any risk factors for atherosclerosis or other vessel disease. Therefore, we believe that the pseudoaneurysm in this patient was the consequence of vessel erosion by the infectious and inflammatory local environment, and for that reason it can be called a mycotic pseudoaneurysm.

When an aneurysm is secondary to vessel infection, the term mycotic aneurysm is often used, as named by Osler in 1885 [4]. However, this term can be misleading, 


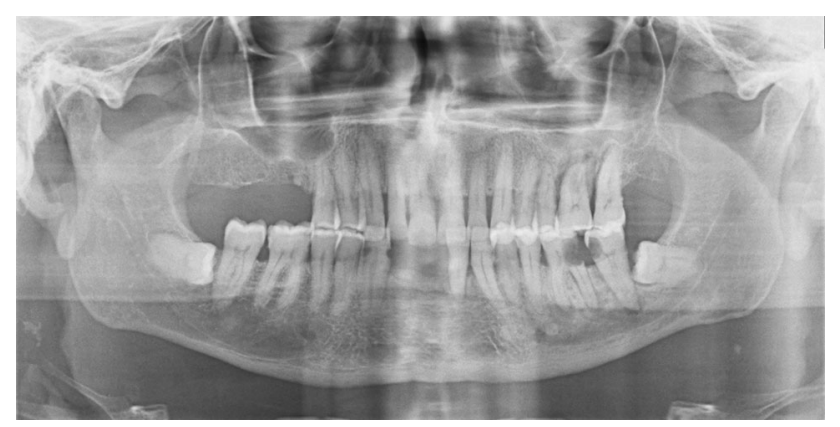

Fig. 5 Case \#2. Panoramic radiograph showing dental caries with periapical osteolysis at the third quadrant

because Osler was describing the mushroom-like appearance, rather than any of the underlying microbiological features. Actually, mycotic aneurysms are more frequently caused by bacterial infection than by fungal infection $[9,10]$.

A PubMed database search of the English literature revealed a few case reports on mycotic true and false aneurysms of the extracranial head and neck vessels. They almost exclusively involved the carotid arteries. A recent review showed a tendency for 20 carotid aneurysm case reports per decade over the last 30 years [11]. Besides the carotid arteries, there is one recent report of an internal maxillary artery mycotic pseudoaneurysm. To the best of our knowledge, there are no previous reports regarding the facial artery. In theory, based on previous descriptions for other head and neck pseudoaneurysms [4], a facial mycotic pseudoaneurysm can pose a threat of sending septic emboli to the brain, more specifically to the cavernous sinus.
In the second clinical case, the etiology is also arguable. The patient was not suitable for contrast imaging at admission and contrast-enhanced CT was only performed after surgery. At that point, it was not possible to ascertain whether the pseudoaneurysm was already concomitant to the odontogenic infection at admission or an iatrogenic result from surgical handling.

These two cases emphasize the importance of careful imaging evaluation of odontogenic infection prior to any invasive procedure. In cases of hemorrhage suspicion, color Doppler ultrasonography can be suggested as a fast screening method, but pain at the swelling site renders it very uncomfortable for the patient and it may not provide sufficient resolution and accuracy, in particular with small vessels. A contrast-enhanced CT scan should be performed whenever possible in head and neck infection, because it allows for abscess identification and may hint at vascular anomalies. Conventional angiography has been the traditional choice for evaluating acute damage to head and neck vessels, but the role of a prior CTA should be further emphasized. Its advantages include noninvasiveness, rapid image rendering, and post-processing capability allowing multiplanar reconstructions that help to confirm the arterial origin of the lesion [10]. Therefore, CTA eases the identification of the parent vessel, and hastens further procedures, particularly a well-planned DSA, with fewer vessels needing to be selected.

The primary therapeutic goal in pseudoaneurysm management is to prevent uncontrolled bleeding. Therefore, we considered that both cases were better managed with DSA embolization. This avoided further unreliable surgical
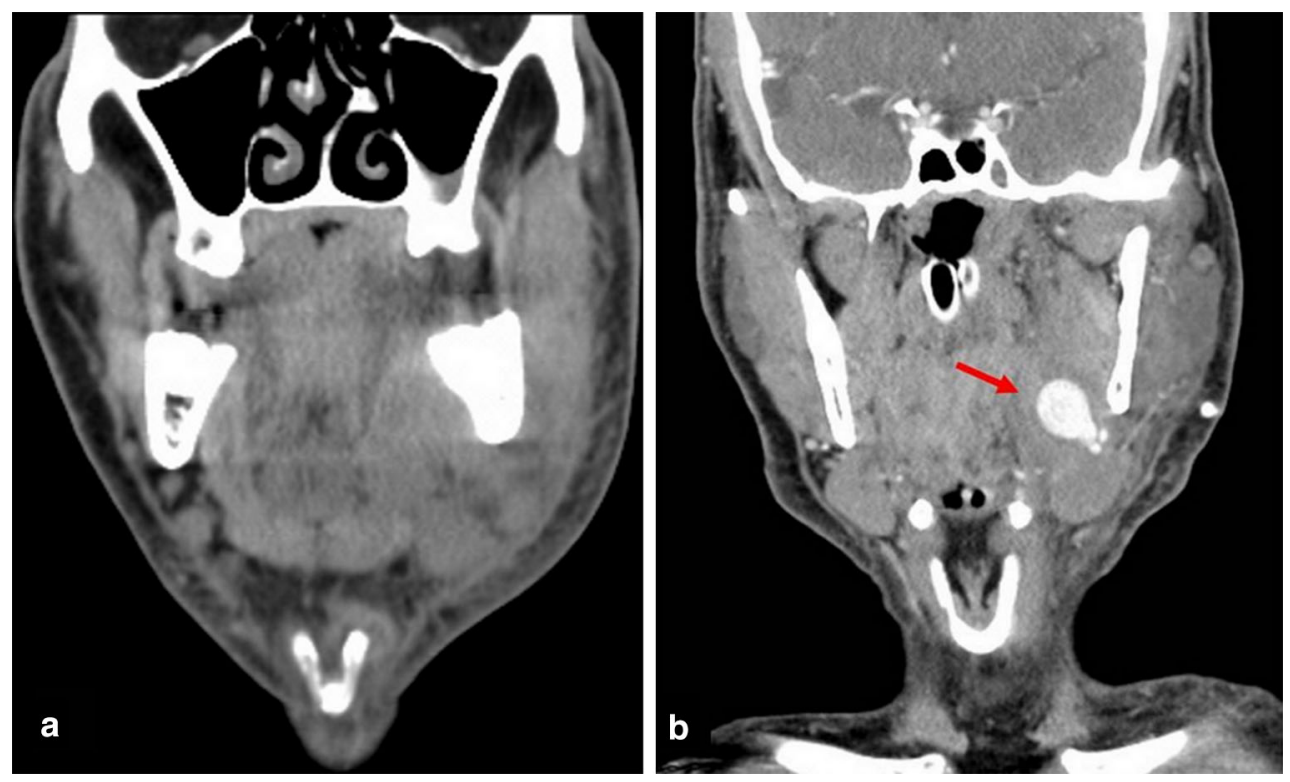

Fig. 6 Case \#2. a Noncontrast CT coronal reconstruction upon patient admission, showing left submandibular enlargement. b Postoperative contrast-enhanced CT coronal reconstruction, showing an enhancing pseudoaneurysm (arrow) in the large mass in the left submandibular area 


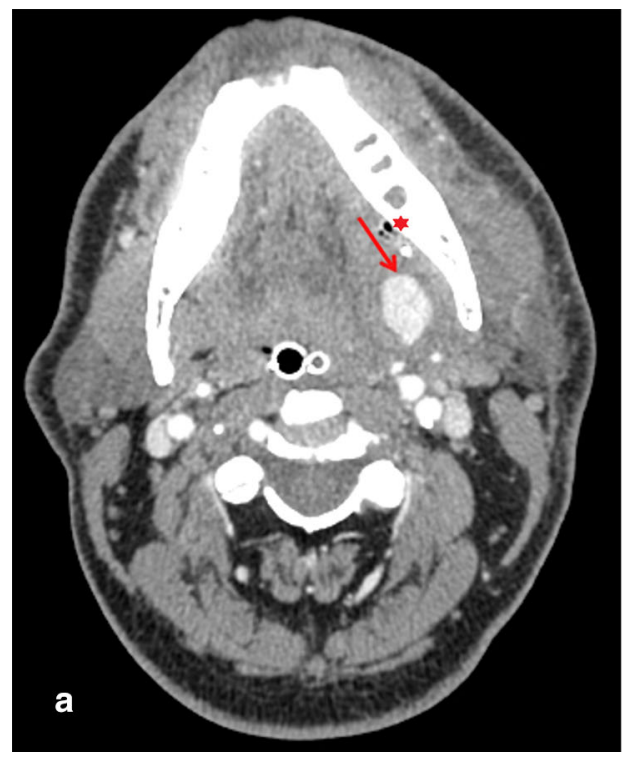

Fig. 7 Case \#2. CTA image showing the pseudoaneurysm (arrow) arising from a branch of the facial artery. a Axial image (source image). There are small gas bubbles close to the left inner surface of

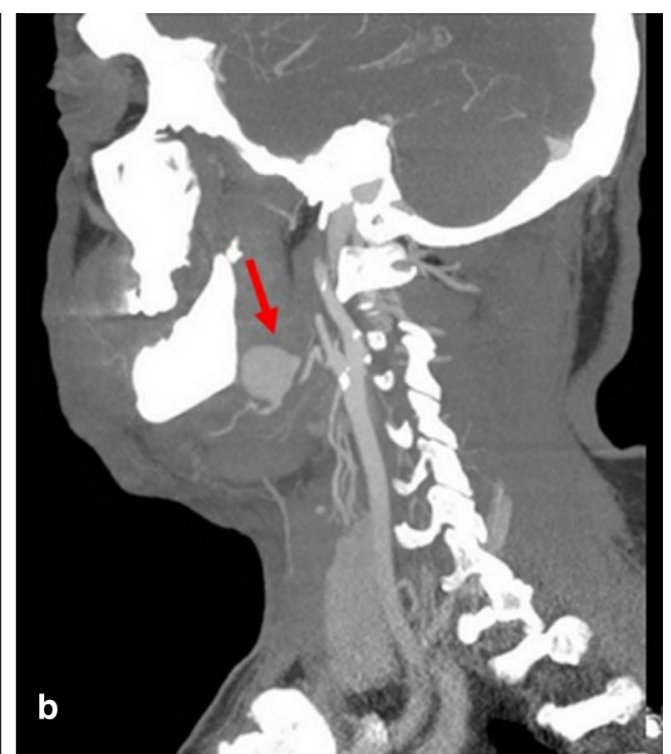

the mandible, suggesting anaerobic bacteria activity (asterisk). b Sagittal image (maximum intensity projection)
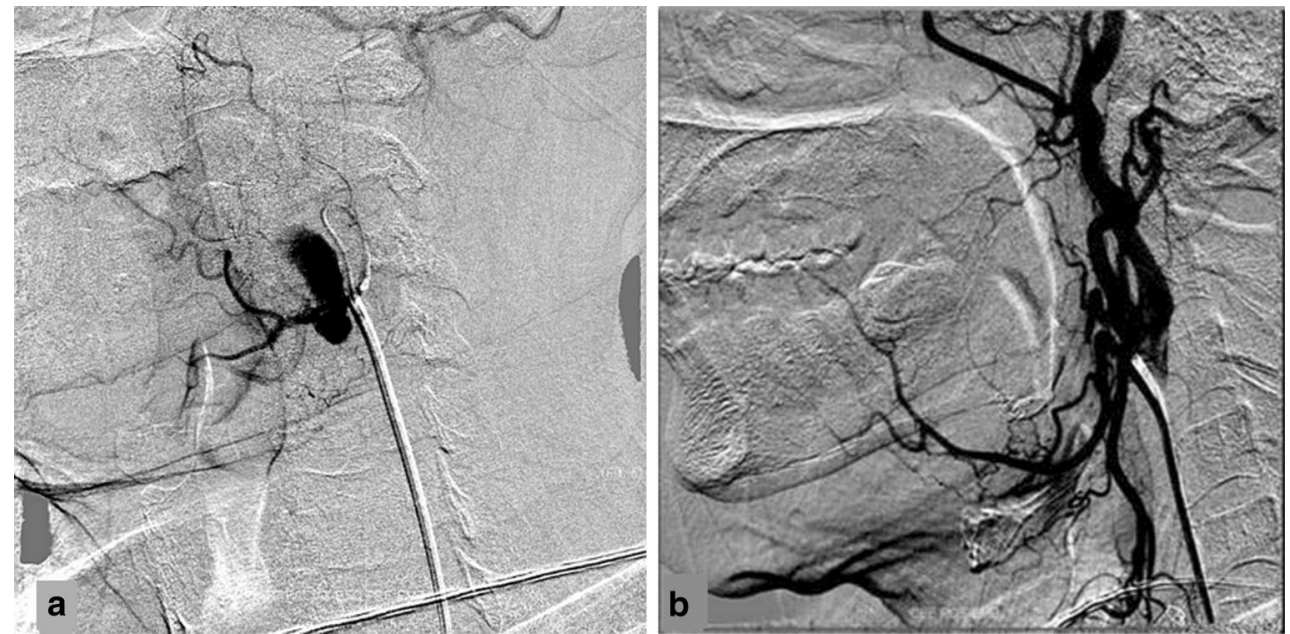

Fig. 8 Case \#2. a Selected DSA image with the microcatheter tip in a proximal facial artery branch, showing a pseudoaneurysm. b DSA image after cyanoacrylate embolization with successful aneurysm exclusion

approaches with the distorted anatomy caused by the infectious swelling, which would require an extensive incision for wide and safe exposure of local blood vessels.

Without a surgical excision, it is not possible to obtain a histopathological assessment to check the vessel wall integrity to yield a definite diagnosis. However, in both cases, the clinical and imaging features were strongly suggestive of pseudoaneurysm.
To summarize, the present clinical cases call attention to a rare but possible vascular consequence of head and neck infections, which can easily be overlooked because of its unusual position, along with the lack of supporting history and clinical findings. A careful imaging choice is essential, because pseudoaneurysms can account for unforeseen bleeding in surgical drainage attempts of head and neck infections. 

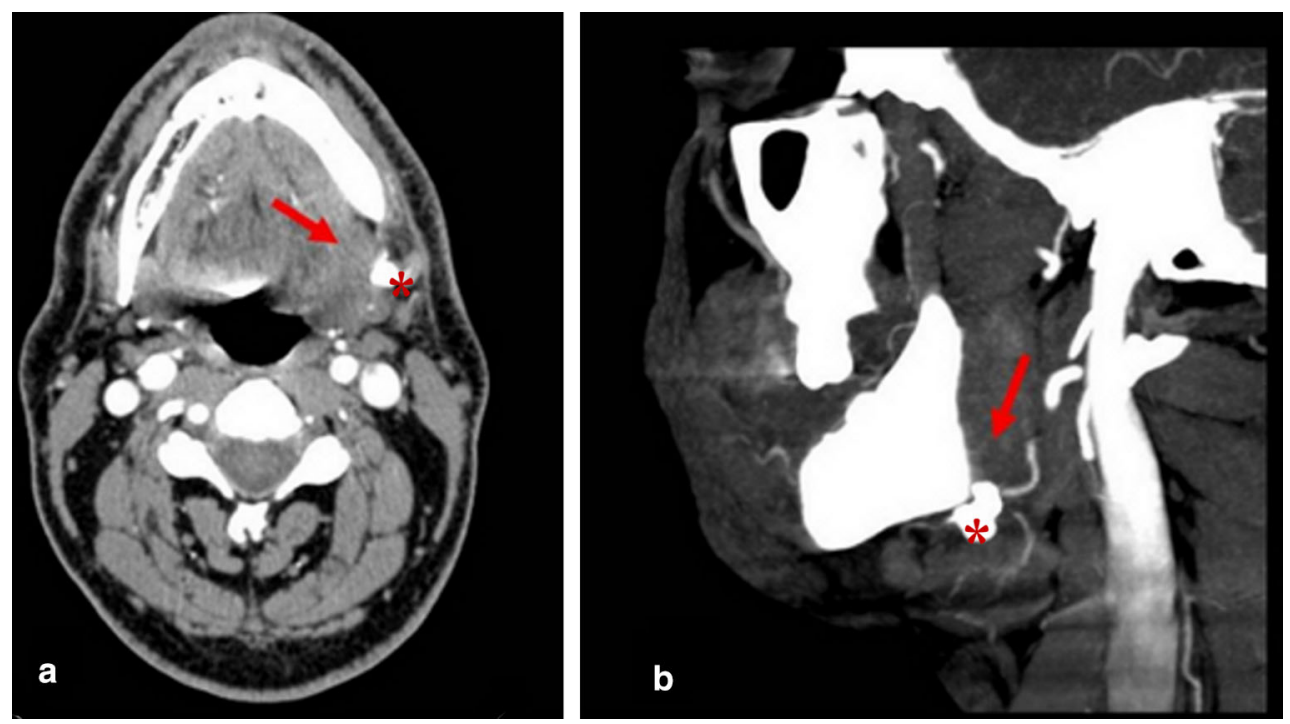

Fig. 9 Case \#2. CTA scans at 1 month after embolization, showing hyperdensity representing the cyanoacrylate (asterisk) with no filling of the pseudoaneurysm (arrow). a Axial image. b Sagittal image

\section{Compliance with ethical standards}

Conflict of interest Miguel Jorge Lopes Oliveira, Carina Marques Pereira Semedo, Maria João Guerreiro Quintela, Isabel Maria Ribeiro Fragata, Carla Cristina Gonçalves Coelho, and Paulo Jorge Valejo Coelho declare that they have no conflict of interest.

Human rights statements and informed consent This article does not contain any studies with human or animal subjects performed by any of the authors. Informed consent was obtained from patients for publication of these case reports and any accompanying images.

\section{References}

1. Hoshi E, Owaki S, Ogawa F, Fukui J, Ogawa T, Shimizu T. True aneurysm of the facial artery. Auris Nasus Larynx. 2010;37:656-8.

2. Ganc D, Prestigiacomo C, Baredes S. Intraparotid arterial aneurysm treated with embolization followed by surgical resection. Ear Nose Throat J. 2012;91:e4-7.

3. Dediol E, Manojlovic S, Biocic J, Franceski D, Ivanac G. Facial artery pseudoaneurysm without evidence of trauma. Int $\mathrm{J}$ Oral Maxillofac Surg. 2011;40:988-90.

4. Pearson S, Choi S. Pseudoaneurysm of the internal carotid artery: a case report and review of the literature. Arch Otolaryngol Head Neck Surg. 2005;131:454-6.

5. Conner WC, Rohrich RJ, Pollock RA. Traumatic aneurysms of the face and temple: a patient report and literature review from 1644 to 1998. Ann Plast Surg. 1998;41:321-6.

6. Rubio-Palau J, Ferrer-Fuertes A, Garcia-Díez E, Garcia-Linares J, Martí-Pagès C, Sieira-Gil R. Traumatic pseudoaneurysm of the superficial temporal artery: case report and review of the literature. Oral Surg Oral Med Oral Pathol Oral Radiol. 2014;117:112-4.

7. Ribeiro-Ribeiro A, Junior S, Pinheiro J. Traumatic pseudoaneurysm of the facial artery: late complication and effects on local blood flow. Oral Surg Oral Med Oral Pathol Oral Radiol Endod. 2011;112:e4-9.

8. Choi H, Kim J, Lee Y, Lee J. Pseudoaneurysm of the facial artery after the injection of local anesthetics. J Craniofac Surg. 2012;23:419-21.

9. Fisk M, Peck LF, Miyagi K, Steward MJ, Lee SF, Macrae MB, et al. Mycotic aneurysms: a case report, clinical review and novel imaging strategy. QJM. 2012;105:181-8.

10. Dunn G, Uppaluri R, Hessler J, Layland M, Derdeyn C, Sunwoo J. Mycotic pseudoaneurysm of the internal maxillary artery. Arch Otolaryngol Head Neck Surg. 2007;133:402-6.

11. Pirvu A, Bouchet C, Garibotti F, Haupert S, Sessa C. Mycotic aneurysm of the internal carotid artery. Ann Vasc Surg. 2013;27:826-30.

12. Rayati F, Parsa H, Abed P, Karagab T. Facial artery pseudoaneurysm following surgical removal of mandibular molar. J Oral Maxillofac Surg. 2010;69:1683-5.

13. Blum C, Theunissen T, Kaplan J. Facial artery pseudoaneurysm after repair of mandibular fracture. J La State Med Soc. 2012;164:194-6.

14. Vieira F, Allen S, Stocks R, Thompson J. Deep neck infection. Otolaryngol Clin North Am. 2008;41:459-83.

15. Rana RS, Moonis G. Head and neck infection and inflammation. Radiol Clin North Am. 2011;49:165-82.

16. Chi T, Tsao Y, Yuan C. Influence of patient age on deep neck infection: clinical etiology and treatment outcome. Otolaryngol Head Neck Surg. 2014;151:586-90.

17. de Lucas EM, Gutiérrez A, Mandly AG, García-Pire F, de Lucas MTM, Parra JA, et al. Life-threatening pseudoaneurysm of the facial artery after dental extraction: successful treatment with emergent endovascular embolization. Oral Surg Oral Med Oral Pathol Oral Radiol Endod. 2008;106:129-32.

18. Jo H, Kim Y, Kang D, Lee S, Kwon T. Pseudoaneurysm of the facial artery occurred after mandibular sagittal split ramus osteotomy. Oral Maxillofac Surg. 2013;17:151-4. 